\title{
An investigation into the possibility of bluetongue virus transmission by transfer of infected ovine embryos
}

\author{
Authors: \\ Estelle H. Venter ${ }^{1}$ \\ Truuske Gerdes ${ }^{2}$ \\ Isabel Wright \\ Johan Terblanche ${ }^{2}$ \\ Affiliations: \\ ${ }^{1}$ Department of Veterinary \\ Tropical Diseases, \\ University of Pretoria, \\ South Africa \\ ${ }^{2}$ Department of \\ Theriogenology, University \\ of Pretoria, South Africa \\ ${ }^{3}$ Virology Section, \\ Onderstepoort Veterinary \\ Institute, South Africa

\section{Correspondence to:} \\ Estelle Venter \\ Email: \\ estelle.venter@up.ac.za

\section{Postal address:} \\ Private bag X04, \\ Onderstepoort 0110 , \\ South Africa \\ Dates: \\ Received: 03 Sept. 2010 \\ Accepted: 16 Nov. 2010 \\ Published: 24 Feb. 2011 \\ How to cite this article: \\ Venter, E.H., Gerdes, T., \\ Wright, I. \& Terblanche, \\ J., 2011, 'An investigation \\ into the possibility \\ of bluetongue virus \\ transmission by transfer of \\ infected ovine embryos', \\ Onderstepoort Journal \\ of Veterinary Research \\ 78(1): Art. \#17, 7 pages. \\ doi:10.4102/ojvr.v78i1.17
}

C 2011. The Authors Licensee: OpenJournals Publishing. This work is licensed under the Creative Commons Attribution License.
Bluetongue (BT), a disease that affects mainly sheep, causes economic losses owing to not only its deleterious effects on animals but also its associated impact on the restriction of movement of livestock and livestock germplasm. The causative agent, bluetongue virus (BTV), can occur in the semen of rams and bulls at the time of peak viraemia and be transferred to a developing foetus. The risk of the transmission of BTV by bovine embryos is negligible if the embryos are washed according to the International Embryo Transfer Society (IETS) protocol. Two experiments were undertaken to determine whether this holds for ovine embryos that had been exposed to BTV. Firstly, the oestrus cycles of 12 ewes were synchronised and the 59 embryos that were obtained were exposed in vitro to BTV-2 and BTV-4 at a dilution of $1 \times 10^{2.88}$ and $1 \times 10^{3.5}$ respectively. In the second experiment, embryos were recovered from sheep at the peak of viraemia. A total of 96 embryos were collected from BTV-infected sheep 21 days after infection. In both experiments half the embryos were washed and treated with trypsin according to the IETS protocol while the remaining embryos were neither washed nor treated. All were tested for the presence of BTV using cell culture techniques. The virus was detected after three passages in BHK-21 cells only in one wash bath in the first experiment and two unwashed embryos exposed to BTV-4 at a titre of $1 \times 10^{3.5}$. No embryos or uterine flush fluids obtained from viraemic donors used in the second experiment were positive for BTV after the standard washing procedure had been followed. The washing procedure of the IETS protocol can thus clear sheep embryos infected with BTV either in vitro or in vivo.

\section{Introduction}

Bluetongue (BT) is a disease that causes substantial economic losses owing to not only its effect on certain species of animals, particularly sheep, but also its associated impact on industries as a result of international regulations restricting the movement of susceptible livestock and their live germplasm. BT is responsible for direct economic losses on affected farms owing to the high morbidity and mortality. Losses in non-endemic regions can be high, with Belgium, for example, having lost a quarter of their sheep population during an outbreak caused by bluetongue virus serotype 8 (BTV-8) in north-western Europe during 2007 (Gloster et al. 2008).

BTV is a member of the genus Orbivirus in the family Reoviridae. BT was first described in South Africa, but has since been recognised in most countries in the tropics and subtropics. Since 1999, however, there have been widespread outbreaks of BT in Greece, Italy, Corsica and the Balearic Islands (Spain). Cases have also occurred in Bulgaria, Croatia, Macedonia, Kosovo and Yugoslavia, which are well north of the generally recognised distribution. It appears that the virus spread to these countries from Turkey and North Africa (Purse et al. 2005; Mellor et al. 2008).

During late September 2000, outbreaks occurred on the Spanish island of Majorca (after an absence of 40 years) and in October on the French island of Corsica. By early December there were additional outbreaks in Majorca and Minorca (Spain): 118 outbreaks involving 14248 sheep in October and subsequently another 166 outbreaks involving 16605 animals (sheep, goats and cattle) also occurred (Purse et al. 2005; Mellor et al. 2008). In October 2006 BTV-8 caused severe outbreaks in Belgium, Italy, Spain and northern European countries in particular (Wilson \& Mellor 2008; Saegerman, Berkvens \& Mellor 2008).

The disease in sheep is characterised by congestion, haemorrhages, cyanosis and ulceration of mucous membranes, particularly those of the buccal cavity. There may be laminitis, myositis and oedema of the head and neck. Some data suggest that foetal abnormalities may occur when ewes are infected early in pregnancy. The severity of the disease varies, with the mortality rate in sheep being between $5 \%$ and $50 \%$. Clinical BT in cattle is rare $(<5 \%$ of infected animals). The virus has little, if any, effect on reproduction, but cattle may develop a prolonged viraemia. Prenatal infection of sheep and cattle foetuses at an early stage of development may lead to embryonic 
death. Foetuses infected during the later stages of gestation survive and develop antibodies, and are not persistently infected (Parsonson 1992).

Transmission of orbiviruses is by Culicoides spp. midges, which are biological vectors. It is thought that the virus can also be transferred from viraemic pregnant dams (sheep and cattle) to their developing foetuses. Although BTV may be found in the semen of some rams and bulls, it can be isolated only at the time of peak viraemia, its presence apparently being the result of infected blood cells in the semen (Osburn 1994). The risk of the transmission of the BTV by bovine embryos does exist, but is negligible if the embryos are washed between collection and freezing and are transported according to the protocol set by the International Embryo Transfer Society (IETS) (Acree et al. 1991; Bowen \& Howard 1984; Thomas, Singh \& Hare 1983, 1985).

Hare et al. (1988) and Singh, Dulac and Henderson (1997) found that BTV-11 was not transmitted when sheep embryos collected from infected donors were washed and transferred to uninfected BT-susceptible recipients. Gilbert, Coubrough and Weiss (1987), however, transferred embryos infected with BTV-10 directly, without washing, to 13 susceptible recipient ewes. None of the ewes became pregnant and nine of them seroconverted. These results support the hypothesis that the zona pellucida of sheep is 'stickier' than that of bovines, which implies that it 'traps' the BTV. This must undoubtedly influence the nature of the washing procedure for the embryos.

There is a constant demand for embryos from South Africa for export to other countries. Figures presented at the 2003 congress of the South African Veterinary Specialist Embryo Group (R. de la Ray) demonstrated the demand for embryos from different animal species. In 1998, 7048 sheep embryos were exported mainly to Australia and Canada, followed by 10408 the next year, and a further 12681 in 2000 to Australia, Canada, China and Brazil. This clearly indicates that South Africa plays a significant role in the world's trade in embryos and that this area can be very viable economically.

Apart from the information mentioned above there is little information on the possible role of sheep embryos in the transmission and spread of BTV and the contradictory results that have been obtained to date create a great deal of uncertainty. IETS has therefore classified BT in sheep as a Category 3 disease (Stringfellow \& Seidel 1998). Although the preliminary evidence indicates that the risk of transmission is negligible, provided that embryos are handled according to protocol, IETS requires additional in vitro and in vivo experimental data to substantiate the preliminary findings mentioned above.

The practical implication for the control of sheep diseases in Category 3 is that countries importing sheep embryos no longer rely only on the procedure of washing the embryos before freezing; additional diagnostic tests are required to be performed on donor animals. This has considerable cost implications for both the exporting and the importing countries. Owing to the requirement for additional diagnostic tests (e.g. determining the BTV status of donors using tissue culture techniques) delivery of consignments of embryos may be delayed considerably.

To reclassify BT from an IETS Category 3 to a Category 1 disease (i.e. one that does not require application of any additional tests) one must be able to certify that embryos are free from BTV and are therefore suitable for export purposes. To achieve this aim two experiments were undertaken (Table 1) to establish (1) whether embryos from BTV-free donors that are infected with BTV in vitro are virus-free after washing according to the IETS protocol, and (2) whether washed and unwashed embryos from BTV-infected donors are virus-free when evaluated for the presence of BTV with an in vitro virus detection (cell culture) method.

\section{Materials and methods Experiment 1}

Twenty BTV-free ewes were purchased and kept in an insectfree animal facility at the Faculty of Veterinary Science, University of Pretoria, Onderstepoort, South Africa. They were confirmed as being negative for BTV antibodies before commencement of the experiment. A commercial groupspecific competitive enzyme-linked immunosorbent assay (C-ELISA) from the Institute for Animal Health, Pirbright was used for this purpose according to the standard operating procedure (SOP) of the Virology Section of the Onderstepoort Veterinary Institute.

A total of 59 ova, embryos or degenerated embryos were collected from 12 of these ewes according to the SOP of the Reproduction Section of the Department of Theriogenology, Faculty of Veterinary Science. The ova and degenerated embryos were separately pooled and infected with BTV-2 or BTV-4 at virus titres of $1 \times 10^{2.88}$ and $1 \times 10^{3.5}$ respectively (Table 2). BTV-infected embryos were incubated for $1 \mathrm{~h}$ at $37{ }^{\circ} \mathrm{C}$ in an atmosphere containing $5 \% \mathrm{CO}_{2}$. Half the number of embryos were then washed using an adapted protocol (Bioniche complete flush medium with Hanks' salts) and treated with trypsin according to the method set out in the

TABLE 1: A summary of the experimental procedure for establishing the infectivity of BTV from in vivo-derived ovine embryos.

\begin{tabular}{ll}
\hline Experiment & Experimental procedure \\
\hline Experiment 1 & - Embryos (approx. 5 per donor) collected from 12 BTV-free \\
& donors \\
& - Exposed 59 embryos to BTV in vitro at a predetermined dilution \\
& Half of the embryos washed and treated with trypsin; half left \\
& unwashed and untreated \\
Experiment 2 & - In vitro assay for BTV in embryos using cell cultures \\
& - Embryos (2-3) collected from 16 BTV-infected donors when \\
& - Virus isolation to prove that donors were infected \\
& - Half of the embryos washed and treated with trypsin; half left \\
& unwashed and untreated \\
& - In vitro assay for BTV in embryos using cell cultures \\
\hline
\end{tabular}


TABLE 2: The number of embryos, ova and degenerated embryos infected with different titres of BTV (Experiment 1).

\begin{tabular}{|c|c|c|c|c|}
\hline \multirow[t]{2}{*}{ Donor number } & \multicolumn{2}{|c|}{ Embryos } & \multicolumn{2}{|c|}{ Ova and degenerated embryos } \\
\hline & $\begin{array}{c}\text { BTV-2 } \\
\text { Titre: } \\
1 \times 10^{2.88}\end{array}$ & $\begin{array}{c}\text { BTV-4 } \\
\text { Titre: } \\
1 \times 10^{3.5}\end{array}$ & $\begin{array}{c}\text { BTV-2 } \\
\text { Titre: } \\
1 \times 10^{2.88}\end{array}$ & $\begin{array}{c}\text { BTV-4 } \\
\text { Titre: } \\
1 \times 10^{3.5}\end{array}$ \\
\hline 15 & - & - & 6 & - \\
\hline 20 & - & - & 5 & - \\
\hline 9 & - & - & - & 2 \\
\hline 34 & - & - & - & 2 \\
\hline 16 & - & - & - & 7 \\
\hline 13 & - & - & 2 & - \\
\hline 2 & - & - & 8 & - \\
\hline 9 & - & 5 & - & - \\
\hline 16 & - & 3 & - & - \\
\hline 2 & - & 2 & - & - \\
\hline 7 & 7 & - & - & - \\
\hline 17 & 3 & - & - & - \\
\hline 17 & - & - & - & 6 \\
\hline 22 & - & - & - & 1 \\
\hline
\end{tabular}

IETS manual (Stringfellow \& Seidel 1998). The washing method was, however, adapted by using micropipette tips from a different manufacturer so that no foreign material could become adherent and so be transferred. The BHK cell culture was also centrifuged and the clear supernatant containing the virus was removed and used as exposure medium. The remaining embryos were neither washed nor treated with trypsin. A BTV isolation method using BHK-21 cells was performed on all the embryos.

Aliquots of wash fluids were taken from wells 1, 2 and 10 in each wash plate, as well as from the media in which the embryos were held before (virus bath) and after washing (holding medium). All the specimens were processed separately on BHK-21 cells.

Embryos were added to $200 \mu \mathrm{L}$ Eagle's minimum essential medium (EMEM) (BioWhittaker, Cambrex) in Eppendorf tubes (AEC-Amersham, Johannesburg), homogenised for $30 \mathrm{~s}$ and centrifuged for $5 \mathrm{~min}$ at $9300 \mathrm{rcf}$ using an Eppendorf 5415 $\mathrm{R}$ centrifuge. Supernatants were aliquoted in 96-well plates in triplicate in $25-\mu \mathrm{L}$ volumes per well. A $100-\mu \mathrm{L}$ volume of BHK-21 cells in EMEM was added and the plates were incubated for 6 days at $37{ }^{\circ} \mathrm{C}$ in an incubator containing $5 \% \mathrm{CO}_{2}$ before reading. The cells were monitored for the cytopathic effects of BTV. Negative cell cultures were blindpassaged after 7 days for two additional passages.

\section{Experiment 2}

The objective of this experiment was to recover embryos from viraemic donors at the peak of viraemia. The same ewes $(n=16)$ as for Experiment 1 were used, together with two rams and two wethers, and were kept in an insect-free animal facility at the Faculty of Veterinary Science. They were again confirmed negative for BTV antibodies by the method as described for Experiment 1 before commencement of the current experiment.
A BTV-4 field isolate, which forms part of the reference collection at the Office International des Epizooties (OIE) reference laboratory (Virology Section, Onderstepoort Veterinary Institute) and is stored in their antigen bank, was used as inoculum. To prepare the material, two sheep received inoculations of $5 \mathrm{~mL}-10 \mathrm{~mL}$ of blood stored in OCG (potassium oxalate, glycerol and water) from a field case. Blood was collected from them in heparin upon developing a febrile reaction (rectal temperature above $40{ }^{\circ} \mathrm{C}$ ) 5-9 days post-inoculation (p.i.). This was used as an inoculum to infect the donors, with approximately $200 \mathrm{~mL}$ providing sufficient fresh, virulent BTV material needed for infection of the ewes. It had a titre of $1 \times 10^{7.8} \mathrm{TCID}_{50}$.

The oestrus cycle of the ewes were synchronised. The ewes were superovulated and inseminated with semen obtained from BTV-negative rams. Each then received an intravenous inoculation comprising $5 \mathrm{~mL}$ of the BTV-4 inoculum. After the first 2 days of inoculation their rectal temperatures were taken twice daily until day 15 p.i.

Reactors were bled for BTV isolation when their rectal temperature reached or exceeded $40{ }^{\circ} \mathrm{C}$ and serum from the donors was assayed for antibodies on days 21 and 28 p.i.

Two or three embryos per donor were collected from the infected ewes 21 days p.i., with a total of 96 being collected. About 40 of the embryos were washed and treated with trypsin according to the techniques prescribed in the IETS manual (Stringfellow \& Seidel 1998), while the others were neither washed nor treated. BTV detection techniques were used on all embryos. Instead of sonication, a vibrating plunger for Eppendorf microcentrifuge tubes was used. Embryos were placed in $200 \mu \mathrm{L}$ EMEM in Eppendorf microcentrifuge tubes and homogenised by a 30-s burst of the vibrating plunger. An individual sterile plunger was used for each specimen.Virus was isolated on BHK cells according to the SOP of the OIE reference laboratory at Onderstepoort. A total of 96 embryos, ova and degenerated embryos were recovered from donor ewes, 7 days after they were inoculated with BTV.

The embryos and their wash fluids as well as the fluids collected from the uterine flushes were cultured directly on BHK-21 cells in 96-well culture plates. Live embryos and degenerated embryos were divided into groups of two or three each, which resulted in a total of 107 specimens being obtained for culture. Each specimen was inoculated on a monolayer in triplicate and three passages were performed before a final result was recorded. Cells were routinely maintained with EMEM supplemented with 5\% foetal calf serum (BioWhittaker-Adcock Ingram, Johannesburg).

To confirm the presence of BTV in the donors, blood samples were collected from them in heparin on the day of flushing. This was processed according to the SOP of the Australian embryo export protocol. Five embryonated chicken eggs were inoculated intravenously per specimen. The eggs were incubated for 7 days at $34.5{ }^{\circ} \mathrm{C}$ during each passage. 
The blood of live embryos in chilled eggs as well as that of recently dead embryos was collected and inoculated into eggs for the second passage. Dead embryos, from either of the egg passages were further subjected to two consecutive 7-day passages on chicken embryo reticulum cells (later reclassified as a hamster cell).

\section{Ethical consideration}

The donors showed a rise in temperature within 4 days after inoculation. They were clinically sick on the day of embryo recovery. Once a temperature of $40{ }^{\circ} \mathrm{C}$ or higher was recorded, the ewes were treated with antibiotics and anti-inflammatory and antipyretic drugs immediately. On the seventh day after inoculation (the day of flushing) all the donors had seroconverted and they tested positive for BTV using a C-ELISA. Classical clinical signs of BT were observed within 4 days after inoculation and some ewes had died.

\section{Results}

\section{Experiment 1}

Results obtained in the investigation where ova, embryos and degenerated embryos that had been infected with BTV

TABLE 3: Results from embryos exposed to BTV in vitro, washed and passaged three times on BHK-21 cells (Experiment 1).

\begin{tabular}{|c|c|c|c|c|}
\hline \multirow[t]{2}{*}{ Sample number } & \multirow[t]{2}{*}{ Sample description } & \multicolumn{3}{|c|}{ Results } \\
\hline & & Passage 1 & Passage 2 & Passage 3 \\
\hline 1 & BTV-2, ova, virus bath & pos & pos & pos \\
\hline 2 & BTV-2, ova, holding media & neg & neg & neg \\
\hline 3 & BTV-2, ova, bath 1 & neg & neg & neg \\
\hline 4 & BTV-2, ova, bath 2 & neg & neg & neg \\
\hline 5 & BTV-2, ova, bath 10 & neg & neg & neg \\
\hline 6 & BTV-2, virus control & pos & pos & pos \\
\hline 7 & BTV-2, embryo, virus bath & pos & pos & pos \\
\hline 8 & BTV-2, embryo, holding media & neg & neg & neg \\
\hline 9 & BTV-2, embryo, bath 1 & neg & neg & neg \\
\hline 10 & BTV-2, embryo, bath 2 & neg & neg & neg \\
\hline 11 & BTV-2, embryo, bath 10 & neg & neg & neg \\
\hline 12 & BTV-4, embryo, virus bath & pos & pos & pos \\
\hline 13 & BTV-4, embryo, holding media & neg & neg & neg \\
\hline 15 & BTV-4, embryo, , bath 2 & neg & neg & neg \\
\hline 16 & BTV-4, embryo, bath 10 & neg & neg & neg \\
\hline 17 & BTV-4, virus control & pos & pos & pos \\
\hline 18 & BTV-4, ova, virus bath & pos & pos & pos \\
\hline 19 & BTV-4, ova, holding media & neg & neg & neg \\
\hline 20 & BTV-4, ova, bath 1 & neg & neg & neg \\
\hline 21 & BTV-4, ova, bath 2 & neg & neg & neg \\
\hline 22 & BTV-4, ova, bath 10 & neg & neg & neg \\
\hline 23 & BTV-2, embryo, virus bath & pos & pos & pos \\
\hline 24 & BTV-2, virus control & neg & pos & pos \\
\hline 25 & BTV-2, embryo, holding media & neg & neg & neg \\
\hline 26 & BTV-2, embryo, bath 1 & neg & neg & neg \\
\hline 27 & BTV-2, embryo, bath 2 & neg & neg & neg \\
\hline 28 & BTV-2, embryo, bath 10 & neg & neg & neg \\
\hline 31 & BTV-4, ova, holding media & neg & neg & neg \\
\hline 32 & BTV-4, ova, bath 1 & neg & neg & neg \\
\hline 33 & BTV-4, ova, bath 2 & neg & neg & neg \\
\hline 34 & BTV-4, ova, bath 10 & neg & neg & neg \\
\hline 35 & BTV-2, ova, unwashed & neg & neg & neg \\
\hline 36 & BTV-4, ova, washed & neg & neg & neg \\
\hline 37 & BTV-4, ova, unwashed & pos & pos & pos \\
\hline 38 & BTV-2, ova, washed & neg & neg & neg \\
\hline 39 & BTV-2, ova, unwashed & neg & neg & neg \\
\hline 40 & BTV-2, ova, washed & neg & neg & neg \\
\hline 41 & BTV-4, embryo, unwashed & neg & pos & pos \\
\hline 42 & BTV-4, embryo, washed & neg & neg & neg \\
\hline 43 & BTV-2, ova, unwashed & neg & neg & neg \\
\hline 44 & BTV-2, ova, washed & neg & neg & neg \\
\hline 45 & BTV-4, embryo, unwashed & neg & neg & neg \\
\hline 46 & BTV-4, embryo, washed & neg & neg & neg \\
\hline
\end{tabular}


in vitro were first exposed to virus and then either washed or left unwashed, followed by passaging three times on BHK cells, are demonstrated in Table 3.

With the exception of three samples, all tested negative for the presence of BTV using BHK-21 cell cultures. BTV was detected in one wash bath (virus passage 3 , bath 1) and two unwashed ova.

\section{Experiment 2}

In this experiment embryos were recovered from viraemic donors at the peak of viraemia, 7 days after infection. Donors were inoculated 7 days before embryo recovery (i.e. on day 14 of the superovulatory programme). Therefore, the day of embryo recovery coincided with the day of peak fever and viraemia. All the donors revealed a rise in rectal temperature $\left(>40{ }^{\circ} \mathrm{C}\right.$ ) from day 4 p.i. and presented with classical signs of BT on the day of embryo flushing.

Although the field isolate induced the development of only mild clinical signs of BT in the two test animals used to prepare the inoculum and were considered not sufficiently severe as to endanger the lives of the experimental animals, the first evidence of virulence came when the first wether (no. 4741) died 9 days after inoculation, while showing severe torticollis. The second wether (no. 4739), however, recovered uneventfully after showing mild clinical signs of BT. The donor ewes were therefore fully susceptible to BT and the inoculated blood induced the disease. Fifteen of them became clinically ill and presented with the classical signs of BT while 12 of them died. One ewe (no. 13) remained unaffected but showed a rectal temperature rise on day 4 p.i. and seroconverted. All the donors had seroconverted by day 7 p.i. Post-mortem examinations revealed the classical lesions of BT, including haemorrhages in the wall of the pulmonary artery, which is regarded as pathognomonic for BT.

A total of 96 live embryos or degenerated embryos were recovered from the donors. All were suitable for use in the trial. Forty-eight were washed while an equivalent number were not washed. BTV was not isolated from any of the embryos, uterine flush fluid or selected wash fluids when specimens of these were cultured directly in tissue culture even after all material had been passaged on BHK cells three times.

The temperature response, clinical signs and tissue lesions observed during post-mortem examination were typical of BTV infection. These, plus the number of chick embryo deaths during intravenous isolation from blood of the donors and the recovery of virus from three spleens prove that these ewes died from BTV infections, the identity of the virus subsequently being confirmed as BTV-4. The vector-free housing in which the animals were kept was also secure.

The antibody titres from the three surviving donor ewes and one wether were determined on days $7,14,21,28$ and 36 p.i. These results are reflected in Table 4 .

\section{Discussion}

Two South African reference strains of BTV, serotypes 2 and 4 , were used to infect live ova and degenerated sheep

TABLE 4: C-ELISA serology results for donors inoculated with BTV and flushed after 7 days (Experiment 2).

\begin{tabular}{|c|c|c|c|c|c|c|c|c|}
\hline \multirow[t]{2}{*}{ Animal Id. number } & \multicolumn{2}{|c|}{ C-ELISA } & \multicolumn{6}{|c|}{ Side-by-side C-ELISA } \\
\hline & Round 1 & Round 2 & Day 0 & Day 0 p.i. & Day 14 p.i. & Day 21 p.i. & Day 28 p.i. & Day 36 p.i. \\
\hline 2 & neg & neg & neg & 51 & + & + & + & + \\
\hline 9 & neg & neg & neg & 64 & + & + & + & + \\
\hline 10 & neg & neg & neg & 60 & + & + & + & + \\
\hline 13 & neg & neg & neg & 52 & 77 & 77 & 79 & 69 \\
\hline 15 & neg & neg & neg & 66 & + & + & + & + \\
\hline 16 & neg & neg & neg & 77 & + & + & + & + \\
\hline 33 & neg & neg & neg & 75 & 78 & 74 & 72 & 74 \\
\hline 34 & neg & neg & neg & 60 & + & + & + & + \\
\hline Ram 4001 & neg & neg & neg & neg & neg & neg & neg & neg \\
\hline Ram 4005 & neg & neg & neg & neg & neg & neg & neg & neg \\
\hline Wether 4741 & neg & neg & + & + & + & + & + & + \\
\hline Wether 4739 & neg & neg & not used & not used & not used & not used & not used & 76 \\
\hline 1 & neg & neg & neg & 77 & + & + & + & + \\
\hline 7 & neg & neg & neg & 70 & 73 & 75 & 74 & 66 \\
\hline 11 & neg & neg & neg & 75 & + & + & + & + \\
\hline 17 & neg & neg & neg & 74 & + & + & + & + \\
\hline 20 & neg & neg & neg & 65 & + & + & + & + \\
\hline 24 & neg & neg & neg & + & + & + & + & + \\
\hline 26 & neg & neg & neg & 59 & + & + & + & + \\
\hline 32 & neg & neg & neg & 36 & + & + & + & + \\
\hline
\end{tabular}


embryos. These strains were selected from the collection held in the BTV OIE reference laboratory at the Onderstepoort Veterinary Institute, because they are well adapted to laboratory conditions and growth in cell culture, well characterised and have a documented history.

The aim of the study was to determine whether the prescribed washing procedure in the IETS protocol is sufficient to 'clean' infected ovine embryos of BTV. Extensive investigations over the past 25 years have provided substantial scientific evidence that bovine embryos derived in vivo from an intact zona pellucida do not transmit disease, including BT, provided that the embryos are properly handled in the period between their collection and transfer (Stringfellow, Givens \& Waldorp 2004). The OIE has adopted the standardised IETS protocol (Stringfellow \& Seidel 1998) for treatment of bovine embryos derived in vivo. Therefore, BT in cattle appears as a Category I disease (caused by a pathogenic agent for which there is sufficient research to show that the risk of transmission via embryos derived in vivo is negligible if the embryos are properly handled between collection and transfer) in Appendix 3 of the OIE manual and the 1998 IETS manual (Stringfellow \& Seidel 1998). Unfortunately, in the case of ovine embryos and BTV, the same conclusion is not possible because there is insufficient published data in this regard. In the 1998 IETS manual (Stringfellow \& Seidel 1998), BT is placed in Category 3, in which the preliminary evidence indicates that the risk of transmission is negligible provided that the embryos are properly handled in the period between their collection and transfer, but for which additional in vitro and in vivo experimental data are required to substantiate the preliminary findings. This was identified in 2004 when Wrathall et al. (2004) proposed that it be changed to Category 2 , that is, a disease for which substantial evidence has accrued to show that the risk of transmission is negligible provided that embryos are handled properly between collection and transfer but for which additional transfers (experimental data) are required to verify the existing data.

In this study, embryos obtained from superovulated ewes were, after infection with BTV, washed and tested for the presence of the virus using a cell culture method. Although the IETS protocol states that the washing procedure is designed to consistently remove $10^{6}-10^{7}$ logs of virus, a pilot trial during which another embryo washing procedure was compared showed the prescribed washing method to be ineffective. The procedure was then slightly modified for use in the first experiment. Firstly, the pipette tips used were changed to consist of an absolutely smooth material so that no material would adhere to them and thus be transferred. Secondly, the infected BHK-21 cell culture was centrifuged and the clear supernatant containing the virus was removed and used as the medium in the bath in which the embryos were exposed to the virus. The results obtained by changing the method were superior to those previously obtained and are illustrated in Table 3. The efficacy of the 10 washes was clearly demonstrated, and the presence of the virus after three passages in BHK-21 cells was only detected in one wash bath and two unwashed embryos exposed to BTV-4 at a titre of
$1 \times 10^{3.5}$. In previous work using a low $\left(1 \times 10^{2.0}\right)$ and a high $\left(1 \mathrm{x} 10^{6.7}\right)$ titre of BTV, the low-titre-infected unwashed embryos were negative, while the high-titre-infected embryos were positive (Terblanche, unpublished information). Clearly the BTV-4 titre used in this study was just above the cut-off limit and resulted in a positive test.

The second objective was to recover embryos from viraemic donors and, after using the IETS wash process, to test for the presence of BTV. The same ewes were used as donors and after synchronisation they were infected with a field isolate of BTV-4. The donors showed a rise in rectal temperature within 4 days of inoculation and embryos were collected from them at the peak of viraemia when they were showing classical clinical signs of BT. Evidence for seroconversion was detected on day 7 p.i. by use of a C-ELISA (Table 4) and the ewes were then flushed. As the ewes showed severe clinical signs they were treated with antibiotics and antiinflammatory and antipyretic drugs once a rectal temperature of $40^{\circ} \mathrm{C}$ or higer was recorded. One ewe died before flushing and 12 of them died within 7 days of surgery. All showed the classical lesions associated with BTV infection on postmortem examination.

All embryos and uterine flush fluids obtained from viraemic donors were negative for BTV after the standard washing procedure had been followed. All the specimens that were collected were cultured directly on cell cultures. In general, in order to improve the sensitivity of the culturing process, field isolates of BTV need to be amplified by growing them in eggs before tissue culture is attempted. It is obvious that in this experiment both the washing and the culturing procedures would have contributed to the uniformly negative result.

There is very little information available on the transmission of BTV via transfer of ovine embryos. The IETS manual, Appendix B (Stringfellow \& Seidel 1998) includes two reports dealing with such transfer of ovine embryos and BTV [i.e. those of Gilbert et al. (1987) and Hare et al. (1988)]. Singh et al. (1997) have also published data on the transfer of the virus via ovine embryos but reported that they had obtained conflicting results. Both Hare et al. (1988) and Singh et al. (1997) found that embryos from BTV-infected donors, when properly washed, were virus-free and could be transferred without infecting recipients. In contrast, they found that when embryos from BTV-free donors were artificially exposed to BTV-11, the virus remained adherent to the embryos, even after washing. Singh et al. (1997) washed embryos according to the IETS protocol, but found that all the inoculated embryonated chicken eggs became infected with BTV, while only five of 11 samples were positive in BHK-21 cells. This clearly illustrates that the culturing procedure employed for BTV isolation is extremely important, unless the virus is well adapted to laboratory conditions.

Gilbert et al. (1987) transmitted BTV-10 to recipient ewes after the embryos that they used had been exposed to the virus for $8 \mathrm{~h}$. They had, however, not used the IETS method for the post-exposure washing procedure, but merely transferred the embryos through four different dishes, each of which 
contained 'clean' medium. After the embryos had been transferred, two of the 15 recipients seroconverted.

\section{Conclusion}

In this report we have shown that sheep embryos infected in vitro with BTV can be cleared of virus by using the washing procedure of the IETS protocol, and that embryos derived from sheep infected with a field strain of BTV were negative for virus after the washing procedure although wild-type virus isolation techniques were not used. This re-emphasises the fact that the risk of transmission of BTV by embryos is negligible if the embryos are handled correctly between collection and transfer.

\section{Acknowledgements}

The research was funded by the National Research Foundation, project no. FA2004051100013. We thank Prof R.C. Tustin for editing the manuscript.

\section{References}

Acree, J.A., Echterkamp, S.E., Kappes, S.M., Luedke, A.J., Holbrook, F.R., PearsonJ.E., et al., 1991, 'Failure of embryos from bluetongue infected cattle to transmit virus to susceptible recipients and offspring', Theriogenology 36, 689-697.

Bowen, R.A. \& Howard, T.H., 1984, 'Transmission of bluetongue virus by intrauterine inoculation or insemination of virus-containing bovine semen', American Journal of Veterinary Research 45, 1386-1388.

Gilbert, R.O., Coubrough, R.I. \& Weiss, K.E., 1987, 'The transmission of bluetongue virus by embryo transfer in sheep', Theriogenology 27, 527-540.

Gloster, J., Burgin, L., Witham, C., Athanassiadou, M. \& Mellor, P.S., 2008, 'Bluetongue in the United Kingdom and northern Europe in 2007 and key issues for 2008', Veterinary Record 162, 298-302.
Hare, W.C., Luedke, A.J., Thomas, F.C., Bowen, R.A., Singh, E.L., Eaglesome, M.D., et al. 1988 , 'Nontransmission of bluetongue virus by embryos from bluetongue virusinfected sheep', American Journal of Veterinary Research 49, 468-472.

Mellor, P.S., Carpenter, S., Harrup, L., Baylis, M. \& Mertens, P.P., 2008, 'Bluetongue in Europe and the Mediterranean Basin: history of occurrence prior to 2006', Preventive Veterinary Medicine 87, 4-20.

Osburn, B.I., 1994, 'The impact of bluetongue virus on reproduction', Comparative Immunology and Microbiology of Infectious Diseases 17, 189-196.

Parsonson, I.M., 1992, 'Overview of bluetongue virus infection in sheep', in T.E. Walton \& B.I. Osburn (eds.), Bluetongue, African Horsesickness and Related Orbiviruses, pp. 713-724, CRC Press, Boca Raton, Florida, USA.

Purse, B.V., Mellor, P.S., Rogers, D.J., Samuel, A.R., Mertens, P.P. \& Baylis, M., 2005 'Climate change and the recent emergence of bluetongue in Europe', Nature Reviews Microbiology 3, 171-181.

Saegerman, C., Berkvens, D. \& Mellor, P.S., 2008, 'Bluetongue epidemiology in the European Union', Emerging Infectious Diseases 14, 539-544.

Singh, E.L., Dulac, G.C. \& Henderson, J.M., 1997, 'Embryo transfer as a means of controlling viral infections. XV. Failure to transmit bluetongue virus through the transfer of embryos from viremic sheep donors', Theriogenology 47, 1205-1214.

Stringfellow, D.A. \& Seidel, S.M., 1998, Manual of the International Embryo Transfer Society, 3rd edn., IETS, Savoy, Illinois, USA.

Stringfellow, D.A., Givens, M.D. \& Waldorp, J.G., 2004, 'Biosecurity issues with current and emerging embryo technologies', Reproduction, Fertility and Development 16 , 93-102.

Thomas, F.C., Singh E.L. \& Hare, W.C.D., 1983, 'Embryo transfer as a means of controlling viral infections. III. Non-transmission of bluetongue virus from viremic cattle', Theriogenology 19, 425-431.

Thomas, F.C., Singh E.L. \& Hare, W.C.D., 1985, 'Embryo transfer as a means of controlling viral infections. VI. Bluetongue virus-free calves from infectious semen', Theriogenology 24, 345-350.

Wilson, A. \& Mellor, P., 2008, 'Bluetongue in Europe: vectors, epidemiology and climate change', Parasitology Research 103, 569-577.

Wrathall, A.E., Simmons, H.A., Bowles, D.J. \& Jones, S., 2004, 'Biosecurity strategies for conserving valuable livestock genetic resources', Reproduction, Fertility and Development 16, 103-112. 